\title{
„Kein Bild, kein Ton? Wir kommen schon.“ - Visuelle Kompetenz im Medienzeitalter
}

\author{
HANS DiETER HUBER
}

Der Beitrag skizziert einige Grundlagenprobleme der gegenwärtigen Bildwissenschaft und versucht, Lösungsansätze dafür zu erarbeiten. Vier Probleme werden diskutiert: 1. Das Problem der „Eigenschaften“ von Bildern, 2. die Probleme symbolischer Repräsentationstheorien von Bildern, 3. die Sozialität von Bildbedeutungen und 4. die künstliche Isolierung des Visuellen von anderen Sinnesinformationen. Es wird der Vorschlag unterbreitet, konnektionistische Bildverarbeitungsmodelle an Stelle von symbolischen Repräsentationstheorien zu verwenden bzw. beide Theorien sinnvoll miteinander zu kombinieren. Im Anschluss daran geht der Beitrag auf die Sozialităt von Bildbedeutungen und Bildkompetenzen ein, die vor allem über den gemeinsam geteilten Sprachgebrauch entstehen. Im Vergleich mit dem Hören wird aufgezeigt, wie problematisch eine methodische Konzentration bildwissenschaftlicher Ansätze auf das rein Visuelle werden kann. Der Beitrag endet mit einem Ausblick auf die Fähigkeit der Selbstanregung des menschlichen Gehirns zur Bildproduktion, hinter der unter den traditionellen Begriffen wie Einbildungskraft oder Imagination die zentralen Wurzeln von Bildkompetenzen zu suchen sind.

The contribution sketches some basic problems of the present image science and tries to offer some moderate solutions for it. Four problems are discussed: 1 . The problem of „properties" of pictures, 2. the problems of symbolic representation theories of pictures, 3 . the sociality of pictorial meaning and 4 . the artificial isolation of visuality from the other senses. It is suggested to use connectionistic models of image processing in place of symbolic representation theories or to combine both theories in a sensible way. Subsequently, the contribution deals with the sociality of pictorial meanings and competences, which are particularly constructed through common linguistic usage. In comparison with hearing, it is pointed out how problematic a methodical concentration of image science on the purely visual can become. The contribution concludes with a short glimpse on the human brain's ability of self-affection for producing images, which is traditionally termed "imagination" and should be seen as the central root of pictorial competences.

\section{Einleitung}

Der Begriff der Kompetenz hat in den letzten zehn Jahren einen enormen Aufschwung erlebt. Im Zusammenhang mit zahlreichen Kopplungen wie „Kompetenzzentrum“, „Schlüsselkompetenz" oder „Kompetenzmanagement" ist er insbesondere in Fragen der Gesellschaftsreform verstärkt verwendet worden. Nichts desto trotz sind die grundsätzlichen Altlasten dieses Konzeptes damit längst nicht beseitigt worden. Man kann die Mode dieses Begriffes an den Erscheinungszahlen von Buchtiteln sehr gut erkennen. Die Deutsche Bibliothek Frankfurt verzeichnet seit 1945 insgesamt 716 Buchtitel, die den Begriff Kompetenz enthalten. Davon sind $74,8 \%$ seit 1990 erschienen; davon wiederum $70,5 \%$ in den letzten 7 Jahren. Während im Jahr 199013 Titel mit dem Titelstichwort „Kompetenz" erschienen sind, waren es 2000 bereits 68 
und im Jahr 200160 Titel.' Die Tendenz ist also besonders in jüngster Zeit stark steigend. Über etwas Selbstverständliches muss man eigentlich nicht reden - und auch nicht schreiben. Denn es ist von selbst verständlich. Wenn also über etwas eigentlich Selbstverständliches eine intensive Diskussion einsetzt, dann kann man fast sicher sein, dass nur noch wenig bzw. gar nichts mehr davon selbstverständlich ist. Bei einer so hohen Zahl von Publikationen zum Thema Kompetenz muss man davon ausgehen, dass wir es seit etwa zehn Jahren mit einer massiven Krise der Kompetenz und mit dem massiven Auftreten von Inkompetenz zu tun haben.

Ich möchte daher einige der strukturellen Kopplungen, in die ein möglicher Begriff von visueller Kompetenz gegenwärtig oder zukünftig situiert werden könnte, ein wenig näher skizzieren und verschiedene Problematiken ansprechen, die hier einer intensiveren Bearbeitung harren.

Zahlreiche Texte über Bilder gehen von der Annahme aus, dass Bilder eigenständige, abgegrenzte Objekte in der Welt „da draußen“ mit bestimmten Eigenschaften seien, die es ermöglichen, sie in notwendiger und hinreichender Weise von anderen Gegenständen der Wẹlt zu unterscheiden. Diese Annahmen gipfeln häufig in der berühmt-berüchtigten Frage „Was ist ein Bild?" Vier verschiedene Problemkreise, die ich näher diskutieren möchte, sind in diesen bisherigen Debatten zu erkennen. Sie behindern meines Erachtens auf entscheidende Weise die Weiterentwicklung der Fragestellung:

1.) Die Behauptung, dass Bilder eigenständige Objekte mit bestimmten Eigenschaften sind,

2.) der Begriff der symbolischen Repräsentation,

3.) die fehlende Berücksichtigung kultureller, sozialer, ökonomischer oder politischer Kontexte beim Gebrauch von Bildern und schließlich

4.) die Isolierung des Visuellen von den anderen Sinnen des kognitiven Systems.

\section{Bilder ohne Eigenschaften}

Auf diese Problematik bin ich an anderer Stelle mehrmals ausführlich eingegangen (siehe HUBER 2001 und HUBER in Vorbereitung) so dass ich hier meine derzeitige Position nur noch einmal kurz zusammenfassen möchte. Kurz gesagt: Bilderfahrung ist weitgehend eine Form

Die Zahlen basieren auf dem Stand des OPAC der Deutschen Bibliothek, Frankfurt/M. am 1.8.2002. Nach 1990 sind 536 Buchtitel von insgesamt 716 erschienen, nach 1995378 Buchtitel.

DIETMAR KAMPER hat in diesem Zusammenhang auf einer gemeinsamen Podiumsdiskussion in Leipzig 1999 im Zusammenhang mit „Kompetenzen“ von einem Kompensationsbegriff gesprochen: „Ich hatte es beiläufig schon angedeutet, daß ich diese Debatte, als sie Ende der sechziger, Anfang der siebziger Jahre im Gang kam, als eine im wesentlichen kompensierende Debatte betrachtet habe. Daß der reale Verlust an Kompetenz in der Gesellschaft in den Theorien zu einer Stilisierung von zugeschriebener Kompetenz führte und in der Theorie noch einmal zu einem Nachleben von Subjektivität, Handlungsfähigkeit, Gefühlssouveränität oder was auch immer man sonst damit verbunden hat. Wenn man da einsteigt, dann ist das natürlich eine Frage der Grundeinschätzung, ob man das für die heutige Zeit noch beanspruchen kann. Wir haben dann danach soviel vom Verlust dieser Grundkompetenzen in den sozialen Verhältnissen gehandelt, bis zum Verschwinden dieses Subjekts, bis zur Auflösung der Identität und der damit konnotierten Fähigkeiten, daß es mir etwas schwer fällt, das für jetzt noch einmal zu reformulieren. Es heißt aber zugleich, daß in dem Versuch einer Historischen Anthropologie der Einsätze der menschlichen Fähigkeiten notwendigerweise auch von den Unfähigkeiten oder von den zunehmenden Unfähigkeiten gesprochen werden muß". (aus Bühler \& Koch 2001, 56/57) 
von Projektion, durch die der jeweilige Beobachter mit Hilfe seines biographischen, lebensweltlichen und gesellschaftlichen Hintergrundes Bedeutungen auf einen materiellen Gegenstand projiziert. Beobachter geben Bildern also ihre Bedeutung, sie nehmen sie nicht von ihnen auf. Die Richtung der Bedeutungszuweisung verläuft nicht von außen nach innen. Bilderfahrung ist keine „Aufnahme“, sondern sie verläuft in weit stärkerem Masse von innen nach außen. Sie ist eine Projektion, die zur Externalisierung oder Objekt-ivierung des Bedeutungsgehaltes führt. Bilderfahrung hat also eine psychische Entlastungsfunktion. Die Bedeutung von Bildern entsteht durch die Projektion von Bedeutungen auf ein Bild. Der projizierte Gehalt wird dann vom Beobachter als die Bedeutung des Bildes ausgegeben. Er wird externalisiert und dem materiellen Objekt als seine Eigenschaft zugeschrieben. Bilderfahrung als ein gesellschaftlich codierter Prozess ist immer eine Projektion, die durch Externalisierung der im sozialen System erzeugten Sinngehalte und deren Zuschreibung an einen materiellen Gegenstand irgendwo in der Welt ,da draußen“" geschieht.

Hinzu kommt, dass Bilder durch den substantivischen Charakter der Sprache, quasi automatisch, als statische Objekte betrachtet werden. Ohne auf den Einfluss des sprachlichen Determinismus auf die Wahrnehmung noch einmal gesondert einzugehen, wie dies BENJAMIN LEE WHORF, VIRGINIA SAPIR und HeLMUT GIPPER eindrücklich gezeigt haben (vgl. Whorf 1963 und GIPPER 1972), möchte ich betonen, dass die Auffassung von Bildern als statischen Objekten mit stabilen Eigenschaften die Probleme erst erzeugt, welche die Bildwissenschaft eigentlich lösen will. Erst seit der Entstehung interaktiver Computer- oder CAVE-Installationen wird nicht nur die temporäre und interaktive Dynamik von Bildern mehr und mehr bewusst, sondern auch die vollständige Abhängigkeit der Bildgenerierung von der Körperbewegung der Beobachter. Im Prinzip versuchten schon der Film und seine mechanischen Vorläufer, die zeitlich eng verknüpfte, temporäre Dynamik des kognitiven Systems zu nutzen, um innere Bewegungen zu erzeugen. So benutzt das Filmbild die Tatsache der Verarbeitungsträgheit des kognitiven Systems zur Erzeugung von Bewegung. Wir wissen aus unser Film-Sozialisation, dass die Bewegungen einer Person in einem Film Schein-Bewegungen sind, die nur im kognitiven System generiert werden und auf dem materiellen Objekt der Filmschicht nicht vorhanden sind. Der mechanische Transport des Filmstreifens erfolgt ruckartig-diskret und wird durch das Malteserkreuz verdeckt. Das Paradoxe ist, dass die sichtbare Bewegung im Film durch eine unsichtbare Bewegung des Films geschieht. Film ist also auf der Ebene des medialen Apparates die Einheit der Differenzen von Stillstand und Austausch, die aber erst beobachtet werden kann, wenn entweder das Malteserkreuz ausgebaut wird oder die Substitution des Bildes und das Verdecken des Bildfensters asynchron erfolgen, z. B. bei Perforationsfehlern.

\section{Die Repräsentation von Bildern}

Damit eng verbunden ist die Frage, wo Bilder existieren. Sind sie draußen in der Welt und mit dem jeweiligen materiellen Objekt identisch oder sind sie es nicht? Meine Auffassung ist die, 
dass Bilder nicht mit dem materiellen Gegenstand, den ich als Bildträger, als Trägersystem oder einfach nur als „Hardware“ bezeichnen möchte, zu verwechseln sind, obwohl sie in der Praxis häufig damit identifiziert werden. Darunter leidet ein großer Teil der Diskussionen über Bilder. Bilder befinden sich immer im kognitiven Systems eines Beobachters. Gleichzeitig sind sie aber auch außen, als materielle Gegenstände. Bilder sind also paradoxale Einheiten, deren Auflösung jeweils nur zugunsten der einen oder anderen Seite betrieben werden kann.

Wenn man bereit ist, zu akzeptieren, dass Bilder intern sind und im kognitiven System vorliegen, dann liegt natürlich die Versuchung nahe, im Gehirn nach einem Ort zu suchen, an dem Bilder repräsentiert und/oder gespeichert werden. Damit sind wir sehr schnell in den Bereich einer weiteren Diskussionen geraten, ob und gegebenenfalls wie Bilder im Gehirn gespeichert und/oder repräsentiert werden, ob es neben der sprachlichen auch eine bildhafte Repräsentationsweise gibt (PAIVIO). Man muss darauf hinweisen, dass der Begriff der symbolischen Repräsentation in diesem Zusammenhang das entscheidende Problem darstellt. Die Kognitionswissenschaft hat das seit längerem erkannt und alternativ versucht, ein Modell von Kognition zu entwickeln, das den problematischen, philosophischen Begriff der symbolischen Repräsentation nicht mehr benötigt oder ihn durch andere Konzepte ergänzt. Unter dem Begriff des Konnektionismus sind diese Ansätze in der Kognitionswissenschaft zu Beginn der achtziger Jahre zusammengefasst worden. Nicht desto trotz geben diese Forschungsansätze, in denen es eher um dynamische, temporäre und hochkomplexe Interaktionen zwischen verschiedenen, räumlich verteilten Gehirnarealen geht als um die Suche nach einem statischen, physikalisch-chemischen Speicherort, wichtige Hinweise für eine mögliche Richtung, in der sich eine zukünftige Bildmedienwissenschaft formieren könnte. Um es kurz zu sagen: Bilder entstehen zunächst nicht durch symbolische Repräsentation, sondern durch dynamische, temporäre Interaktionen zwischen einfachen, räumlich verteilten Komponenten.

Konnektionistische Vorstellungen des kognitiven Funktionierens unseres Gehirns stellen eine Alternativlösung zu der gebräuchlichen Auffassung von Kognition als einer Art Informationsverarbeitung mit Hilfe symbolischer Repräsentationen dar. Beide Modelle, das repräsentationale und das konnektionistische, wurden in den Ursprüngen der Kognitionswissenschaft zu Beginn der fünfziger Jahre in den USA auf den Macy Conferences als Alternativen vorgetragen, während im weiteren Verlauf die Repräsentationstheorie der Kognition eindeutig zum vorherrschenden Paradigma wurde. Mitte der achtziger, Anfang der 90er Jahre entdeckte man im Zusammenhang mit neuen Untersuchungsmethoden der Neurophysiologie, der Neurobiologie und der Informatik diese Modelle auch wieder aufs Neue. Konnektionistische Modelle der Kognition beruhen unter anderem auf dem Versuch, unser Wissen über die neuronale Funktionsweise des Gehirns in Modelle des kognitiven Funktionierens einfließen zu lassen.

Konnektionistische Modelle setzen anstelle einer lokalisierten Symbolverarbeitung und Speicherung verteilte Vorgänge an, die sich über ein ganzes Netzwerk von einzelnen Komponenten erstrecken und globale Eigenschaften hervortreten lassen. Das Gehirn arbeitet offensichtlich auf der Basis massiv verteilter Wechselwirkungen. Denn die Nachteile der symbo- 
lisch-repräsentationalen Informationsverarbeitung bestehen darin, dass sie auf nacheinander angewandten Regeln basieren. Zweitens ist Symbolverarbeitung lokalisiert, d. h., sie ist sehr störanfällig. Verteilte, parallele Verarbeitungsprozesse sind deshalb sehr wünschenswert, weil sie eine relative Gleichwertigkeit und Immunität gegenüber Störeinflüssen gewährleisten. In einem Netzwerk entsteht eine globale Kooperation, die spontan in Erscheinung tritt. Konnektionistische Theorien liefern elegante Arbeitsmodelle für viele interessante kognitive Fähigkeiten wie Schnellerkennung, assoziative Erinnerung und begriffliche Generalisierung. In konnektionistischen Systemen sind die Bedeutungselemente keine symbolischen Repräsentationen, sondern komplexe, dynamische Muster des Austausches zwischen den vielen Einheiten, die das Netzwerk bilden. Im konnektionistischen Ansatz ist Bedeutung eine Funktion des globalen Systemzustandes. Er entsteht aus einem Netzwerk von Einheiten, die einfacher und subtiler sind als Symbole. Man spricht deshalb auch von einem subsymbolischen Paradigma (VARELA, THOMPSON \& Rosch 1995, 143; siehe auch SMOLENSKy 1988).

So wissen wir z. B., dass das Gehirn das Prinzip der unspezifischen Reizcodierung verwendet. Das heißt, dass keine qualitativen Unterschiede von Neuron zu Neuron übertragen werden, sondern nur quantitative, nämlich das Feuern oder Nicht-Feuern von Synapsen. Das Interessante ist dabei, dass unser Gehirn jedoch nicht wie ein Computer zu funktionieren scheint. Während das Rechnen komplizierter Zahlenfolgen einem Computer leichter und uns eher schwerer fällt, gelingt uns ein schnelles Wiedererkennen in komplexen Situationen, wie z. B. auf einer Cocktailparty, besonders leicht, während ein Computer hier mit einem Mustererkennungsverfahren besonders lang brauchen würde. Wir sind offensichtlich bei parallelen Verarbeitungsweisen sehr schnell, während ein mühseliges, sequentielles Abarbeiten schneller vom Computer erledigt wird.

Als wichtigste Vorteile konnektionistischer Systeme werden deren Robustheit und Lernfähigkeit genannt. Gerade bei Bildverarbeitungsprozessen ergeben sich auch in der Computersimulation neuronaler Netzwerke bedeutende Vorteile gegenüber einer sequentiellen Verarbeitung (vgl. DIEBNER \& SAHLE 2001). Sie liegen in der massiven Parallelität der Datenverarbeitung. Konnektionistische Systeme besitzen aber auch Nachteile. Diese machen sich dann bemerkbar, wenn man versucht, sequentielle Prozesse oder Abläufe zu modellieren, die hochgradig sequentiell oder sukzessiv sind.

VARELA, THOMPSON \& ROSCH haben den interessanten Vorschlag unterbreitet, subsymbolische Emergenz in einem Netzwerk und symbolische Repräsentation in Beziehung zueinander zu setzen. Sie schlagen vor, die Anfangsphase des Sehens bis zur primären Sehrinde konnektionistisch zu erklären, dann bei der unteren Windung des Schläfenlappens auf symbolische Programme umzuschalten (VARELA, THOMPSON \& ROSCH 1995). Dieser Vorschlag würde sehr gut mit der Annahme eines der Gründungsväter der Kognitiven Psychologie, ULRIC NEISSER, zusammenpassen, der bereits 1967 folgendes mutmaßte: 
Von den konstruktiven Vorgängen wird angenommen, daß sie zwei Stufen haben, deren eine schnell, grob, ganzheitlich und parallel ist, während die andere bewußt, aufmerksam, detailliert und sequentiell ist. (NEISSER 1974, 27)

Das könnte die enorme Schnelligkeit erklären, mit der wir in der Lage sind, selbst hochkomplexe Situationen sehr effizient zu verarbeiten. Ein konnektionistisches Netzwerk könnte also in einer schnellen und einfachen Parallelität die ersten Gruppierungen wie Gestaltbildung, Figur-Hintergrund-Unterscheidungen und Teil-Ganzes-Verhältnisse erzeugen. Hinzutreten könnten dann sequentielle, auf symbolischer Repräsentation beruhender Prozesse, die langsam, mühselig und bewusst ablaufen. In einer Kombination von konnektionistischer und repräsentationaler Reizverarbeitung würden sich bestimmte Phänomene der visuellen Beobachtung ebenfalls besser erklären lassen. Deswegen geht man gegenwärtig in der Kognitionswissenschaft davon aus, dass beide Modelle zur Erklärung sensorischer Reizverarbeitung geeignet sind.

Wir würden daher sehr viel gewinnen, wenn wir uns von den beiden Dogmen der symbolischen Repräsentation und der Auffassung von Bildern als statischen Objekten verabschieden würden. Wenn wir Bilder als komplexe zeitliche Ereignisse oder schnelle Folgen kurzer, räumlich und zeitlich verteilter Ereignisse statt als statische, räumliche Objekte betrachten, würde vieles deutlicher sichtbar werden. Dass Bilder in der Zeit entstehen, dass Zeit nötig ist, um materielle Gegenstände visuell zu erfassen, dass visuelle Beobachtung ein zeitlicher dynamischer Vorgang ist, alles dies ist hinreichend lange bekannt. Die Einsicht, dass die Konstruktion von Bildern im kognitiven System ein komplexes, dynamisches System bildet, das nur temporär besteht und in aufeinander folgenden und gleichzeitigen Interaktionen zwischen räumlich verteilten Gehirnarealen besteht, ist dagegen weniger populär. Einen entscheidenden Anteil spielt natürlich hier das Gedächtnis bzw. welche Auffassung von der Funktion des Gedächtnisses wir haben. Es betrifft Fragen der Erinnerung, der Identifizierung, des Lernens und des daraus folgenden Handelns. Für eine Theorie der Bildkompetenz ist sie daher die zentrale Instanz. Es würde sich daher lohnen, einen Ausflug in die Geschichte der Gedächtnisforschung zu unternehmen, um zu sehen, welche nicht-repräsentationalen Modelle wir analog zu konnektionistischen Netzwerken zur Verfügung haben, um die Fragen von Lernen, Üben, Können, Erinnern und Vergessen im Zusammenhang mit der visuellen Beobachtung von Bildmedien $z u$ thematisieren.

\section{Die Sozialität der Kognition}

Noch ein Wort zum Begriff der Kognition. Kognition wird meistens als individuell und an einen kognizierenden Organismus gebunden aufgefasst. Von daher wird sie auch gerne dem Sozialen, Politischen, Gesellschaftlichen oder Kulturellen gegenübergestellt, wie man das Individuelle dem Kollektiven und das Private dem Öffentlichen gegenüberstellt. Mit einer individualpsychologischen Auffassung von Kognition kommt man aber auch in der Bilderfrage nicht weiter. Vielmehr ist in dasjenige, was wir beispielsweise einen individuellen kognitiven 
Stil in der visuellen Beobachtung nennen, das Soziale, Gesellschaftliche, Politische, Ökonomische und Kulturelle einer bestimmten Zeit, einer bestimmten Kultur, einer bestimmten Sozialisation und einer bestimmten Klassenzugehörigkeit längst schon eingegangen. Noch bevor ein Beobachter irgendeine Beobachtung macht, ist das, was er beobachten kann, immer schon sozial, gesellschaftlich, politisch, ökonomisch und kulturell vorgeformt. Am Einfachsten können wir die soziale Überformtheit oder Bedingtheit von Kognitionen an Gewohnheiten, Erwartungen, und Vorurteilen erkennen. Kognitionen sind deshalb konventionell, sie sind von kulturellen, sozialen und politischen Schemata überformt. Dass das Denken frei sei oder das Beobachten von Bildern ein individuelles Erlebnis, ist ein Mythos, der eine ganz bestimmte ideologische Funktion in der Gesellschaft erfüllt.

Wie kommen wir also von individuell verkörperten Kognitionen zu sozialer Bedeutung? Für gewöhnlich wird hier auf die Sprache verwiesen. Gesprochene oder geschriebene Sprache, als eine kollektive, gesellschaftliche Struktur, die immer schon vorhanden ist, ist für viele die soziale Schicht der Kommunikation per se. So spricht HuMBERTo MATURANA von languaging, vom In-der-Sprache-Sein, das uns eigentlich erst zu sozialen Wesen machen würde (vgl. MAturana 1998, 362). Auch hier kann man wieder die einseitige Bevorzugung diskursiver Systeme beobachten. Wenn es stimmt, dass die Sprache die Sozialität unserer Beobachtungen, unseres Handelns und unserer Existenz garantiert, dann gilt dieses Argument in gleicher Weise auch von Bildern. Auch in Bildern treffen wir auf die hoch konventionalisierte, gesellschaftliche Zurichtung eines Mediums. Bilder funktionieren immer schon und ausschließlich, noch vor ihrer Herstellung, in einem hoch konventionalisierten sozialen Kontext, in dem von vorneherein genauestens bestimmt ist, was als ein gelungenes Bild zählt und wie es zu funktionieren hat. Man kann sogar noch weiter verallgemeinern: Von jedem öffentlich beobachtbaren Medium gilt, dass es ein Dispositiv, eine Zurichtungsapparatur für die Konventionalität unserer Beobachtungen ist. Indem wir uns dem medialen Apparat von Big Brother unterwerfen, werden wir zu ,freien“ und vor allem ,,individuellen“ Subjekten.

Nicht nur die Struktur der Sprache oder unseres in der Sprache-Seins (languaging) garantieren die Sozialität unserer Bedeutungen, sondern auch alle anderen potentiell öffentlich beobachtbaren und zugänglichen Medien. In jedem Medium unserer Gesellschaft finden wir immer schon, noch vor jeder Artikulation und jeder konkreten Beobachtung, die angestellt wird, eine gesellschaftliche, politische, kulturelle und soziale Struktur vor, die in jeder Hinsicht generalisiert und konventionalisiert ist. Für potentiell öffentlich beobachtbare Bilder bedeutet das, dass ihre Zirkulation in der Gesellschaft die Sozialität, Konventionalität und Geteiltheit unserer Erfahrungen garantiert und strukturiert. Indem wir uns also der sozialen, politischen, gesellschaftlichen und kulturellen Strukturiertheit der Medien unterwerfen, werden wir in dieser Unterwerfung zu Subjekten. Die Medien versprechen, uns zu freien Subjekten und einzigartigen Individuen zu machen. Das ist der Deal, den die Gesellschaft der Lüge mit uns abschließt. Dafür dürfen wir dann die Medien benutzen. Wir empfinden, fühlen, und den- 
ken entlang der Strukturdispositive des Sozialmediums und sonst wahrscheinlich nicht viel mehr.

Das Schwierige für Künstler besteht nun gerade darin, sich von der Kulturalität, Sozialität und Konventionalität der medialen Apparate zu lösen, und Möglichkeiten zu entdecken, die zwar vorhanden sind, aber als nicht genutzte Ressourcen bisher übersehen wurden. Hier kann speziell der künstlerische Umgang mit Bildern in einem sehr weiten Sinne die Machtstruktur der Unterwerfung aufbrechen und durch die Produktion neuer, innovativer Möglichkeiten die Dispositive der Unterwerfung und der Subjekt-ivierung in Frage stellen.

\section{Die Isolierung des Visuellen}

Ich komme zu dem vierten Problem, der Isolierung der visuellen Wahrnehmung von den anderen Sinnen und den daraus resultierenden Folgen. Wenn man sich einen Grossteil der Wahrnehmungsforschung der letzten 50 Jahre ansieht, findet man eine fast skandalös anmutende, einseitige Konzentration auf visuelle Wahrnehmung und vor allem individualpsychologische Ansätze, Methoden und Experimente. Bestenfalls findet man noch Ausführungen zur akustischen Wahrnehmung. Aber man kann Regale von Büchern über Wahrnehmung durchblättern. Kaum etwas findet man über Riechen, Fühlen und Schmecken. Um in der Bildproblematik weiterzukommen, halte ich es jedoch für zwingend nötig, die gesamte Bandbreite an sinnlichen Beobachtungsmöglichkeiten zu berücksichtigen. Es leuchtet, vom Visuellen her gesehen, nicht unbedingt ein, den Fokus hier weiter aufzuziehen. Ich werde daher als didaktischen Kunstgriff einen Umweg über das Hören und andere Sinne machen, um unseren visuellen Sinn besser in diesem Gesamtfeld sinnlicher Orientierungsmöglichkeiten situieren zu können. Ein zweites Manko kommt hinzu. Ein Grossteil der Forschung über visuelle Beobachtung findet unter individualpsychologischen Prämissen statt. Fast jede Wahrnehmungstheorie, die man antreffen kann, vernachlässigt die sozialen, politischen und kulturellen Voraussetzungen, Kontexte und Einflüsse auf die scheinbar so freie und individuelle Wahrnehmung.

Visuelle Beobachtung ist stets und immer begleitet von anderen Sinneswahrnehmungen, wie Hören, Riechen, Fühlen, Schmecken und Tasten sowie propriozeptiven Eigenwahrnehmungen. Visuelle Wahrnehmung ist darüber hinaus stets und immer verkörpert. Sie geschieht mit und in einem Körper, der sich in Raum und Zeit umherbewegen kann. Sie ist letzten Endes eine körperliche und biologische Aktivität, nämlich verkörpertes Handeln (vgl. VARELA, THOMPSON \& ROSCH 1995). Jede Außenwahrnehmung ist begleitet von propriozeptiven Eigenwahrnehmungen. Beobachtung besteht immer aus diesen beiden Bestandteilen: Heterozeption und Propriozeption, Aufmerksamkeit für sich selbst und Aufmerksamkeit für die Welt. Erst die Möglichkeit zur Propriozeption gestattet es einem Beobachter, den Unterschied zwischen sich selbst und dem anderen als solchen, nämlich als einen Unterschied, der Sinn macht, zu beobachten. Erst die Möglichkeit, sich selbst zu beobachten, ist die Voraussetzung 
für die Möglichkeit, etwas anderes als etwas anderes zu beobachten, sprich eines Anderen als eines Außen. Diese Unterscheidung kann jedoch selbst nur als eine Unterscheidung im Inneren eines kognitiven Systems getroffen werden.

Um die Leistungen kognitiver Systeme bei der Generierung von inneren oder äußeren Bildern besser verstehen zu können, bietet sich ein kleiner Umweg an. Wie bereits gesagt, führt die Isolierung der Wahrnehmungsforschung auf visuelle Beobachtung zu einem sehr eingeschränkten und künstlichen Verständnis von Bildwahrnehmung. Über den Umweg über das Hören, das Tasten, das Riechen und die Geschmackswahrnehmung können wir zahlreiche scheinbare Selbstverständlichkeiten im visuellen Beobachten entdecken, aber auch auf Blindheiten aufmerksam machen und so erneut für eine Wissenschaft vom Bilde sensibilisieren.

Stellen wir also beispielsweise analog zur Frage „Gibt es ,innere’ Bilder?“ die Frage „Gibt es ,innere' Geräusche?“ Wo ist der Ort des Hörens oder des Gehörten? Man könnte dieselbe Frage für das Schmecken, das Riechen und das Tasten stellen. Wo ist der Ort des Schmeckens? Wo ist der Ort des Tastens? Wo ist der Ort des Riechens? Alle diese Fragen haben ein und dieselbe Antwort. Der Ort, von dem die Rede ist, befindet sich im kognitiven System. Aber eben mit der bedeutenden Einschränkung, dass sich das kognitive System immer schon gemäss den politischen, sozialen und kulturellen Dispositiven der medialen Apparate strukturiert, also selbst bereits gemäss den Prämissen sensorischer Politiken funktioniert.

Wo ist also der Ort des Gehörten? Es ist nicht der Ort, an dem sich das gehörte Ereignis ereignet, sondern der Ort, an dem man es hört. Der Ort des Hörens ist immer nur dort, wo jemand hört. Er ist niemals woanders. Es gibt zwar ein Ereignis, das durch seine Bewegung Schallwellen auslösen kann. Nehmen wir als Beispiel einen Blitz und als sein akustisches Pendant den Donner. Wir hören aber nicht das Schallwellen verursachende Ereignis, sondern die Schallwellen selbst, und zwar dann und nur dann, wenn sie auf unser Trommelfell auftreffen. Wir neigen dann dazu, folgendermaßen zu antworten: „Ich habe den Donner gehört" und nicht „Ich habe plötzlich starke Druckschwankungen in meinem Ohr registriert“. Auch hier lässt sich sofort die Tendenz beobachten, den im eigenen kognitiven System erfahrenen Sinnesreiz wieder nach außen zurück zu verlagern, ihn also zu externalisieren und dadurch zu objekt-ivieren. Die Objektivierung stellt jedoch ein entscheidendes Hindernis für ein angemessenes Verstehen des Vorgangs auditiver Kognition dar. Denn es gibt viele schallwellenverursachende Ereignisse, die wir aus der Ferne sehen, aber aus Gründen der Windrichtung nicht hören können. Das Hören ist immer innen, wenn so sagen will, es findet immer hinter dem Trommelfall statt, nicht davor. Andererseits wird es sofort wieder an ein externes Ereignis oder Objekt in der Welt, da draußen' zurückgerechnet. Hier kann man deutlich das Motiv erkennen, das hinter solchen schnellen Externalisierungen steht. Es ist der Wunsch, sich stets neu in einer ,objektiven' Welt zu verankern, sich ihr zu unterwerfen, um an sie zu glauben, als wäre sie das authentische, wahrhaftige Eine. Nach jeder Veränderung des Aktivitätszustandes eines kognitiven Systems wird die strukturelle Kopplung, die den Organismus in jedem Moment seiner Existenz mit einer ihn umgebenden Umwelt verbindet, neu stabilisiert. 
Beim Hören ist es interessant, dass wir zwar ebenfalls wie beim Sehen einen vergleichbaren Oberbegriff wie „Bild“ kennen, nämlich „Geräusch“. Aber „Geräusch“ suggeriert im Gegensatz zu „Bild“ kein statisches Objekt oder einen räumlich abgegrenzten Gegenstand, sondern eine zeitliche Sequenz, eine Abfolge von kurzen Ereignissen mit einer gewissen Dauer, einem Anfang und einem Ende. Die Fähigkeit zur Selektion, zum bewussten Hinhören und Herausfiltern spezifischer Geräusche aus einem diffusen Umfeld, dem kognitiven Ausblenden des medialen Hintergrundrauschens gibt es auch in der visuellen Wahrnehmung. Es scheint wohl generell so zu sein, dass dieselben Mechanismen von positiver und negativer Selektion, von Gruppierung, Gestaltbildung und Abgrenzung für alle menschlichen Sinne in gleichem Masse gelten. Das würde nicht für eine kanalspezifische Verarbeitung sprechen, sondern gerade im Gegenteil für kanal- oder reizunspezifische Verarbeitungsmechanismen des kognitiven Systems.

Das Problem bei der Beobachtung von Bildern ist der gewohnheitsmäßige Hang zur Objekt-ivierung und Externalisierung. Wenn man stattdessen versuchen würde, Bilder als zeitliche Ereignisse oder als kurze Folgen mit einer bestimmten Dauer, mit Anfang und Ende, zu betrachten, käme man in dieser hartnäckig vertrackten Frage vielleicht etwas weiter. Dann würde nämlich deutlich werden, dass Bilder im Allgemeinen keine statischen Entitäten oder Objekte sind, wie der substantivische Gebrauch des Wortes nahe legt, sondern eher schnelle Abfolgen von kurzen Ereignissen, die das kognitive System in Aktivität versetzen. Ferner würde deutlich werden, dass statische Bilder nur ein Sonderfall von Bildern sind, auf den sich allerdings aus historischen, kulturellen und politischen Gründen in hohem Maße unsere bisherige Aufmerksamkeit gerichtet hat. Es sind sozusagen Bilder, die stillhalten, damit man sie in Ruhe beobachten kann.

\section{Innere Bilder und visuelle Kompetenz}

Wo ist also der Ort der Bilder? Wo befinden sich Bilder?, frage ich noch einmal. Es gibt keine gegebenen Bilder und es gibt auch keine unmittelbare Wahrnehmung gegebener Bilder. Beobachtung ist immer medial vermittelt. Sie geschieht durch ein Medium, und zwar durch das Medium des Sehens. Bilder sind auch nicht mit bestimmten materiellen Objekten da draußen in der Welt identisch. Bilder können nur im kognitiven System eines Beobachters existieren.

Wenn man dieses Argument als Ausgangspunkt einer Debatte über visuelle Kompetenz akzeptieren will, kann man einige interessante Phänomene erklären. Das menschliche Gehirn ist nämlich durchaus in der Lage, sich selbst anzuregen und in seiner Eigentätigkeit selbständig Bilder hervorzubringen. In der Alltagssprache bezeichnen wir diese Fähigkeit zur Simulation mit Begriffen wie Phantasie, Vorstellungsvermögen, Imagination, Träumen, etc. Interessanterweise sind diese Vorstellungsbilder meist bewegt und nicht statisch. Wir können uns eine bestimmte Szenerie vorstellen, z. B. mit zwei Personen, die wir gut kennen, und wir können diese Personen in unserem kognitiven System willentlich dazu veranlassen, sich durch 
den Raum und die Zeit zu bewegen, die wir ihnen zugewiesen haben. Wir können uns ihre Kleidung vorstellen, ihre Stimme, ihre Bewegung, den Raum, in dem sie sich umherbewegen, vielleicht sogar ihren Geruch. Wir können sie jetzt z. B. veranlassen, sich mit dem rechten Arm durchs Haar zu fahren. Das alles stellt kein Problem dar. Es gelingt auf Anhieb. Die Szenerie ist durchaus farbig und mehr oder weniger reich differenziert. Die Vorstellungsbilder sind farbig, räumlich, sie bewegen sich in der Zeit und sind auch mit Hör- und Geruchseindrücken verbunden. Dehnt man diesen Gedanken weiter aus, müsste man konsequenterweise davon ausgehen, dass das kognitive System durch Selbstanregung in der Lage ist, Geschmacksvorstellungen, Geruchs-, Tast- und propriozeptive Szenarien zu entwickeln, bewusst zu erleben und willentlich ablaufen zu lassen. Hier kommen wir nun an den entscheidenden Punkt. Exakt diese Fähigkeit zur Selbstanregung des Gehirn ist der Ursprung von visueller Kompetenz.

Eine visuelle Vorstellung kann also sowohl durch eigene Imaginationstätigkeit als auch durch eine von außen angeregte visuelle Beobachtung hervorgerufen werden. $\sqrt{\text { Visuelles Beo- }}$ bachten ist immer eine Kombination aus beidem, aus Selbstanregung und Fremdanregung, aus Simulation und Stimulation. Die inneren, durch Selbstanregung entstandenen Vorstellungsbilder sind eine wichtige Grundlage und Quelle künstlerischer und nicht-künstlerischer Bildproduktion.

Generell kann man für jedes beliebige Medium die Behauptung aufstellen, dass Übung, Erfahrung und Begabung die Grundlage für die Externalisierung innerer Bilder in einer öffentlich beobachtbaren, visuellen Form bilden. Damit wir von ihrer Existenz erfahren können, ist die Medialisierung dieser Vorstellungen in einem öffentlich zugänglichen Bildangebot notwendig. Hier beginnt Medienkompetenz und im engeren Sinne Bildkompetenz. Sie beginnt bei der Fähigkeit, visuelle Vorstellungen in einem potentiell öffentlich beobachtbaren Medium formulieren zu können. Im Prinzip ist jedes Medium zur Inszenierung visueller Vorstellungen mehr oder weniger geeignet. Man kann visuelle Vorstellungen beschreiben, zeichnen, malen, plastisch formen, installieren, tanzen, mit seinem Körper ausdrücken, performen, auf einem Musikinstrument vorspielen, mit der Videokamera darstellen oder als Website konstruieren.

Zu dieser visuellen Kompetenz, die darin besteht, Bilder in einem öffentlich beobachtbaren Medium erzeugen zu können, kommt eine zweite hinzu, nämlich diejenige, Bilder lesen und verstehen zu können. Dass es sich hierbei ebenfalls um eine aktive, konstruktive, kulturelle, soziale und politische Fähigkeit handelt, die in kultureller Sozialisation erworben werden muss, ist beim Blick auf fremde Kulturen und ihre visuellen Systeme leichter beobachtbar als bei der Beobachtung der eigenen visuellen Kultur. Schwierigkeiten beim Lesen fremder Bildsysteme treten häufiger bei bildlichen Darstellungen anderer Kulturen auf. Innerhalb unserer eigenen westlich-christlichen Mediensozialisation erscheinen uns die gebräuchlichsten Darstellungskonventionen als so selbstverständlich, dass wir sie gar nicht mehr als konventionell codiert begreifen, sondern sie uns als objektiv und realistisch erscheinen. Um innere Bilder in 
einem öffentlich zugänglichen Medium formulieren zu können, sind also ebenso Kenntnisse und Fertigkeiten notwendig wie bei der Fähigkeit, diese lesen und verstehen zu können. Die Frage stellt sich hier, ob diese Kompetenzen völlig verschieden sind, ob sie sich gegenseitig überlappen oder ob sie gar identisch sind.

\section{Literatur}

BÜHLER, MARCEl \& Koch, AleXANDeR (2001) (Hg.): Kunst \& Interkontextualität. Materialien zum Symposium , schau-vogel-schau“, Köln: Salon-Verlag.

Diebner Hans H. \& SaHle, Sven (2001): On the Role of the Micro-Macro-Transition and Control Processes for Understanding the Interface, in: DiEBNER, HaNS H., Druckrey, Timothy \& Weibel, Peter (Hg.): Sciences of the Interface, Tübingen: Genista Verlag, 261-271.

GIPPER, HeLMUT (1972): Gibt es ein sprachliches Relativitätsprinzip?, Frankfurt a. M.: Fischer.

HUBER, HANS DIETER (im Druck): „Wir können nicht verstehen, was wir nicht verstehen können." Konstruktivistische Aspekte des Verstehens von Bildern, in: BERING, KUNIBERT (Hg.): Kunstvermittlung. Perspektiven einer Didaktik der Bildenden Künste (Jahreshefte der Kunstakademie Düsseldorf 5), Düsseldorf.

HUBER, HANS DIETER (2001): Irritierende Bilder. Wie verstehen wir, was wir sehen? in: SACHS-HOMBACH, KLAUS (Hg.): Bildhandeln. Interdisziplinäre Forschungen zur Pragmatik bildhafter Darstellungsformen (Reihe Bildwissenschaft Bd.3), Magdeburg: Scriptum Verlag.

MAturana, Humberto M. (1998): Biologie der Realität, Frankfurt a. M.: Suhrkamp.

NEISSER, UlRIC (1974): Kognitive Psychologie, Stuttgart: Klett Verlag.

SMOLENSKY, PAUL (1988): On the proper treatment of connectionism; in: Behaviour and Brain Sciences 11, 1-74.

WhORF, BENJAMIN LEE (1963): Sprache, Denken, Wirklichkeit. Beiträge zur Metalinguistik und Sprachphilosophie, Reinbek: Rowohlt.

VAREla, Francisco J., ThOMPSOn, Evan \& Rosch Eleanor (1995): Der mittlere Weg der Erkenntnis. Der Brückenschlag zwischen wissenschaflicher Theorie und menschlicher Erfahrung, München: Goldmann. 